\title{
Unparticle Effects on Unitarity Constraints from Higgs Boson Scattering
}

\author{
Xiao-Gang He and Chung-Cheng Wen \\ Department of Physics and Center for Theoretical Sciences, \\ National Taiwan University, Taipei, Taiwan, R.O.C.
}

(Dated: November 18, 2018)

\begin{abstract}
We study the effects of two-body Higgs boson scattering by exchanging unparticles. The unparticle contribution can change the standard model prediction for two-body Higgs boson scattering partial wave amplitude significantly leading to modification of the unitarity constraint on the standard model Higgs boson mass. For unparticle dimension $d_{\mathcal{U}}$ between 1 and 2, the unitarity constraint on Higgs boson mass can be larger than that in the SM. Information on unparticle interaction can also be obtained.

PACS numbers: 12.15.-y, 12.60.Fr, 11.80.Et, 14.80.Cp
\end{abstract}


Since the seminal work of Georgi on unparticle physics 11 last year, the study of unparticle effects has drown a lot of attentions [1, 2, 3, 4, 5, 6, 7, 8, 9, 10, 11, 12]. The concept of unparticle [1] stems from the observation that certain high energy theory with a nontrivial infrared fixed-point at some scale $\Lambda_{\mathcal{U}}$ may develop a scale-invariant degree of freedom below the scale. The kinematics is determined by its scaling dimension $d_{\mathcal{U}}$ under scale transformations. The unparticle must interact with Standard Model (SM) particles to be physically relevant. Even though at present the detailed dynamics of how unparticle interacts with SM particles is not known, these interactions can well be described in effective field theory. In this approach the interactions are parameterized in the following way [1]

$$
\lambda \Lambda_{\mathcal{U}}^{4-d_{S M}-d_{\mathcal{U}}} O_{S M} O_{\mathcal{U}}
$$

where $O_{S M}$ is composed of the SM fields, and $O_{\mathcal{U}}$ is an unparticle operator.

There has been a burst of activities on various aspects of unparticle physics from phenomenology to theoretical issues [1, 2, 3, 4, 5, ㄷ, 7, 8, 9, 10, 11, 12]. Some of the major tasks of phenomenological study are to search for new signals and effects in various physical processes, and to determine (constrain) the unparticle scale and also unparticle dimension $d_{\mathcal{U}}$. In this work we study unparticle interaction effects on unitarity constrains from two-body Higgs boson scattering using partial wave analysis. We find that the unparticle contribution to the scattering partial wave amplitude can be significant which affect the unitarity constraint on the Higgs boson mass. For $d_{\mathcal{U}}$ between 1 and 2, the unparticle contribution can relax the upper bound for Higgs boson mass.

Partial wave analysis of scattering processes is one of the often used methods to constrain unknown parameters in a theory. The unitarity constraint on Higgs boson mass from twobody Higgs boson scattering in the SM[13], and constraint on extra dimension scale[14] are some of the interesting examples. A scattering amplitude $\mathcal{M}$ for a given process can be decomposed into partial wave amplitude according to angular momentum $\vec{J}$ as

$$
\mathcal{M}=\frac{1}{k} \sum a_{J}(2 J+1) P_{J}(\cos \theta) .
$$

The unitarity condition is referred to the condition that the magnitude for each of the partial wave amplitude $\left|a_{J}\right|$ should not be too large. There are many discussions of how to implement unitarity condition to constrain new physics[15]. We will use a weak condition $\left|a_{J}\right|<1$ for $J=0$ and work with the tree level amplitude to show how interesting constraints on unparticle interactions and Higgs boson mass can be obtained. 

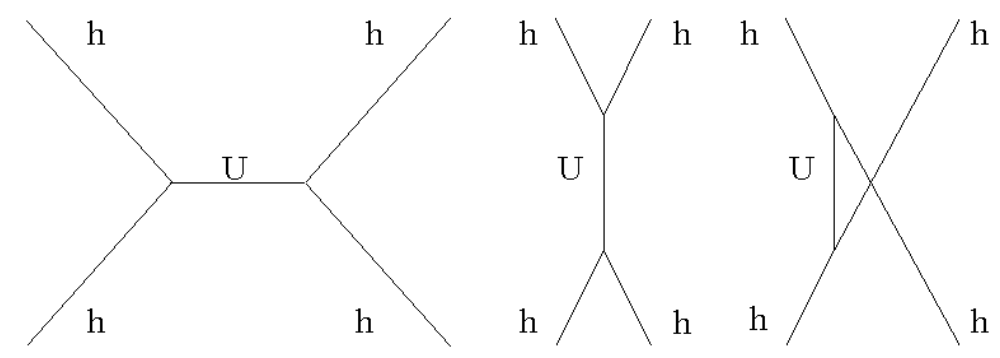

FIG. 1: Feynman diagrams for two-body Higgs boson scattering by exchanging an unparticle in $s$, $t$, and $u$ channels.

Potentially large contribution to the two-body Higgs boson scattering may come from the following lowest dimension operator involving a scalar unparticle and SM Higgs field[11, 12]

$$
O_{h h}=\lambda_{h h} \Lambda_{\mathcal{U}}^{2-d_{\mathcal{U}}} H^{\dagger} H O_{\mathcal{U}}
$$

where $H=\left(h^{+},(v+h+i I) / \sqrt{2}\right)$ is the SM Higgs doublet. The $h^{+}$and $I$ are the fields "eaten" by $W$ and $Z$, and $h$ is a physical Higgs. The parameter $\lambda_{h h}$ is real.

There are $s, t$ and $u$ channel contributions from the above effective operator to the two-body Higgs boson scattering amplitude as shown in Fig. 1. We obtain the scattering amplitude as

$$
\mathcal{M}^{u n}(h h \rightarrow h h)=\left(\lambda_{h h} \Lambda_{\mathcal{U}}^{2-d_{\mathcal{U}}}\right)^{2} \frac{A_{d_{\mathcal{U}}}}{2 \sin \left(\pi d_{\mathcal{U}}\right)}\left[\frac{1}{(-s)^{d_{\mathcal{U}}}}+\frac{1}{(-t)^{d_{\mathcal{U}}}}+\frac{1}{(-u)^{d_{\mathcal{U}}}}\right]
$$

In obtaining the above expression, we have used the scalar unparticle propagator $\left(i A_{d_{\mathcal{U}}} / 2 \sin \left(\pi d_{\mathcal{U}}\right)\right)\left(1 /\left(-p^{2}\right)^{2-d_{\mathcal{U}}}\right)$. The factor $A_{d_{\mathcal{U}}}$ is normalized as $A_{d_{\mathcal{U}}}=$ $\left(16 \pi^{5 / 2} /(2 \pi)^{2 d_{\mathcal{U}}}\right) \Gamma\left(d_{\mathcal{U}}+1 / 2\right) /\left(\Gamma\left(d_{\mathcal{U}}-1\right) \Gamma\left(2 d_{\mathcal{U}}\right)\right)$ following ref.[1].

Using the above scattering amplitude, the $\mathrm{J}=0$ component in the partial wave expansion $a_{0}^{u n}$ can be easily obtained

$$
\begin{aligned}
a_{0}^{u n} & =\frac{1}{16 \pi}\left(\frac{4 \vec{p}^{2}}{s}\right)^{1 / 2} \frac{1}{s-4 m_{h}^{2}} \int_{-\left(s-4 m_{h}^{2}\right)}^{0} \mathcal{M}^{u n} d t \\
& =\frac{1}{16 \pi} \lambda_{h h}^{2}\left(\frac{\sqrt{s}}{\Lambda_{\mathcal{U}}}\right)^{2 d_{\mathcal{U}}-4} \frac{A_{d_{\mathcal{U}}}}{2 \sin \left(\pi d_{\mathcal{U}}\right)} \sqrt{1-\frac{4 m_{h}^{2}}{s}}\left[e^{-i \pi d_{\mathcal{U}}}+\frac{2}{d_{\mathcal{U}}-1}\left(1-\frac{4 m_{h}^{2}}{s}\right)^{d_{\mathcal{U}}-2}\right],
\end{aligned}
$$

where $\vec{p}=\sqrt{1-4 m_{h}^{2} / s}$ is the Higgs boson momentum in the center of mass frame.

As for any other processes involving unparticle propagator, there is a $\sin \left(\pi d_{\mathcal{U}}\right)$ factor in the denominator which has poles at integer $d_{\mathcal{U}}$ and makes $a_{0}^{u n}$ to diverge. In the first term in eq.(5), the pole at $d_{\mathcal{U}}=1$ is cancelled by a zero in $A_{\mathcal{U}}$. However, the second term will 


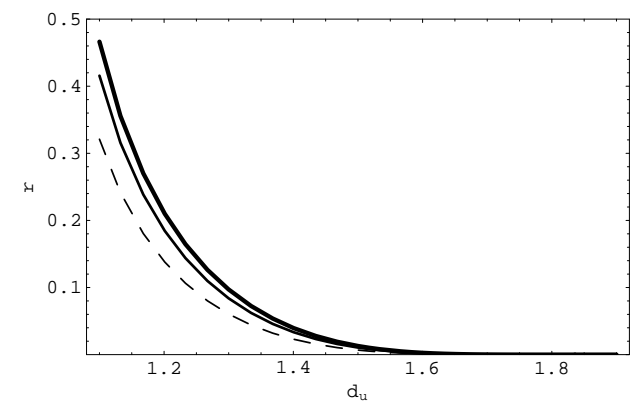

FIG. 2: Lower bound on $r$ as a function of $d_{\mathcal{U}}$ with different Higgs masses, 115 (solid line), 500 (lighter solid line), 1000 (dashed line) GeV in the limit $s>>4 m_{h}^{2}$.

blow off. Therefore integer numbers are forbidden. Also the factor $A_{d_{\mathcal{U}}}$ decreases quickly as $d_{\mathcal{U}}$ increases, therefore for large $d_{\mathcal{U}}$ the unparticle contribution is suppress.

For a complete analysis, one also needs to include the SM contribution where the $J=0$ partial wave amplitude is given by [13]

$$
a_{0}^{S M}=\frac{G_{F} m_{h}^{2}}{8 \sqrt{2} \pi} \sqrt{1-\frac{4 m_{h}^{2}}{s}}\left[3+\frac{9 m_{h}^{2}}{s-m_{h}^{2}}-\frac{18 m_{h}^{2}}{s-4 m_{h}^{2}} \ln \left(\frac{s}{m_{h}^{2}}-3\right)\right] .
$$

With this contribution included, the weak unitarity condition becomes

$$
\left|a_{0}^{T}\right|=\left|a_{0}^{S M}+a_{0}^{u n}\right|<1
$$

There is an imaginary part from unparticle contribution to $a_{0}$ due to the s-channel unparticle exchange in Fig. 1 with $\operatorname{Ima}_{0}^{u n}=-(1 / 32 \pi) r^{2 d_{\mathcal{U}}-4} A_{d_{\mathcal{U}}} \sqrt{1-4 m_{h}^{2} / s}$. Here $r=\left(\lambda_{h h}\right)^{1 /\left(d_{\mathcal{U}}-2\right)} \sqrt{s} / \Lambda_{\mathcal{U}}$. Since the SM contribution is real, the unitarity condition requires $\left|I m a_{0}\right|<1$. One can, in principle, obtain a constraint on the parameter $r$ as a function of the unparticle dimension. We have analyzed this and found that the constraints are weak. The combined effects of real and imaginary parts can provide more interesting information which we study in the following.

In the limiting case of $s>>4 m_{h}^{2}$, weak unitarity condition is simply given by,

$$
\left|r^{2 d_{\mathcal{U}}-4} \frac{A_{d_{\mathcal{U}}}}{32 \pi \sin \left(\pi d_{\mathcal{U}}\right)}\left[e^{-i \pi d_{\mathcal{U}}}+\frac{2}{d_{\mathcal{U}}-1}\right]+\frac{3 G_{F} m_{h}^{2}}{8 \sqrt{2} \pi}\right|<1 .
$$

Since the strength of the unparticle contribution to the partial wave amplitude is a function of $r$, the unitarity condition may provide information about $r$. We plot, in Fig. 2, $r$ as a function of $d_{\mathcal{U}}$ for several representative Higgs boson masses for $d_{\mathcal{U}}$ in the range between 1 and 2. Note that the unitarity bound gives a lower bound for $r$ because $2 d_{\mathcal{U}}-4<0$. 
This reflects the fact that the interaction of Higgs boson with unparticle defined by operator $O_{h h}$ does not decouple in the limit $\Lambda_{\mathcal{U}}$ goes to infinite. Since $r \sim \sqrt{s} / \Lambda_{\mathcal{U}}$, naively, for $d_{\mathcal{U}}$ smaller than 2, small $s$ is ruled out. However, one must keep in mind that $s>4 m_{h}^{2}$ must be satisfied, $s$ smaller than $4 m_{h}^{2}$ is not constrained by unitarity condition. For $d>2$, the unitarity bound gives a upper bound for $r$. In this case, in the large $\Lambda_{\mathcal{U}}$ limit, the interaction of Higgs boson and unparticle decouples.

If the unparticle scale $\Lambda_{\mathcal{U}}$ is known from some theoretical considerations, one can use the weak unitarity condition to constrain the energy scale $\sqrt{s}$ with which one can reliably (satisfying the weak unitarity condition) use the operator $O_{h h}$ for calculations. We have carried out a study keeping $4 m_{h}^{2} / s$ term in the expression for $a_{0}^{T}$. For $d_{\mathcal{U}}$ larger than 2 , the unitarity condition enables one to obtain an upper bound for $s$ since the leading $s$ dependence is $s^{d_{\mathcal{U}}-2} . s$ cannot be too large in order not to violate the unitarity condition, but numerically it is way above $10 \mathrm{TeV}$ or any near future collider energies, such as LHC and ILC. For $d_{\mathcal{U}}$ between 1 and 2, the unitarity condition puts a low bound for $s$. Since the leading scale $\Lambda_{\mathcal{U}}$ and $s$ dependence of $a_{0}^{T}$ is $\left(s / \Lambda_{\mathcal{U}}^{2}\right)^{d_{\mathcal{U}}-2}$, the unitarity condition gives a lower bound for $s$. A smaller $\Lambda_{\mathcal{U}}$ corresponds to a larger $s$. Numerically we find that for lower values of $\Lambda_{\mathcal{U}}$ (less than $1 \mathrm{TeV}$ ), s larger than the threshold is all allowed. But for larger $\Lambda_{\mathcal{U}}$, for example $10 \mathrm{TeV}$, there are regions with $d_{\mathcal{U}}$ close to 1 violate unitarity condition for $s$ above the threshold. Note also that near the threshold, the second term in eq.(5) become very large and therefore $\left|a_{0}^{T}\right|$, if $d_{\mathcal{U}}$ is smaller than 1.5. One should not use value for s too close to the threshold if $d_{\mathcal{U}}$ is less than 1.5.

We find that the weak unitarity condition is satisfied for $s$ significantly larger than the threshold of producing two Higgs bosons for $d_{\mathcal{U}}$ between 1 and 2 .

We now discuss unparticle effects on unitarity constraint on Higgs boson mass $m_{h}$. Without unparticle contribution, in the limit $s>>4 m_{h}^{2}$, the weak unitary condition implies that the Higgs boson mass must be smaller than $8 \sqrt{2} \pi / 3 G_{F}=1010 \mathrm{GeV}$. With unparticle contributions, the constraint on Higgs mass can be modified dramatically since the real part of the unparticle contribution can have either signs relative to the SM contribution depending on the unparticle dimension $d_{\mathcal{U}}$. For example, for $d_{\mathcal{U}}$ between 1 and 2, the real part of $a^{u n}$ is negative making the constraint on Higgs mass looser compared the one for SM. For $d_{\mathcal{U}}$ between 2 and $3, \operatorname{Re}\left(a^{u n}\right)$ is positive, the constraint on Higgs mass becomes tighter. Since for large $d_{\mathcal{U}}$, there is a suppression from $A_{d_{\mathcal{U}}}$, the constraint on relevant parameters are weak. 

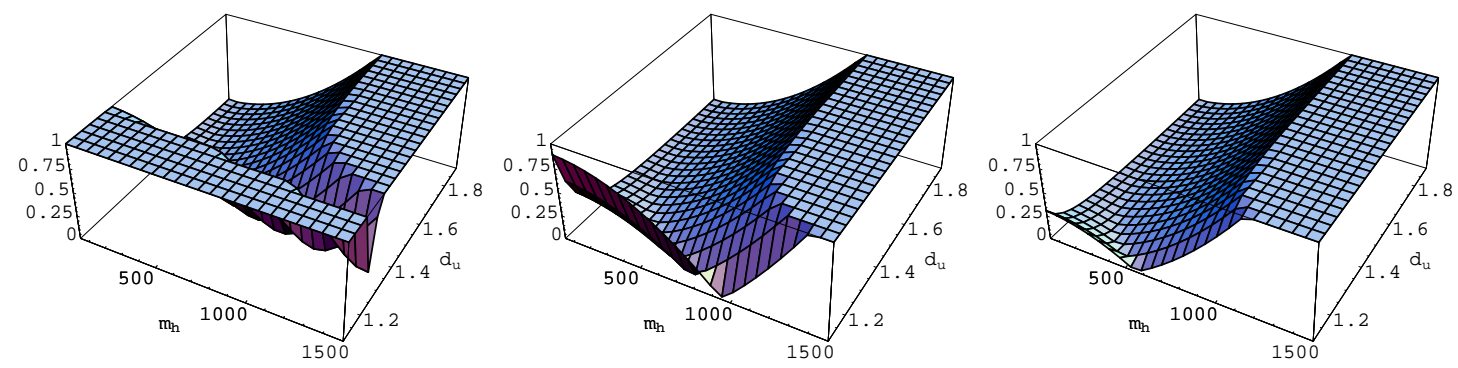

FIG. 3: $\left|a_{t}^{0}\right|$ (vertical axis) as a function of Higgs mass( in GeV) and $d_{\mathcal{U}}$ with $r=0.1,0.5$ and 1.

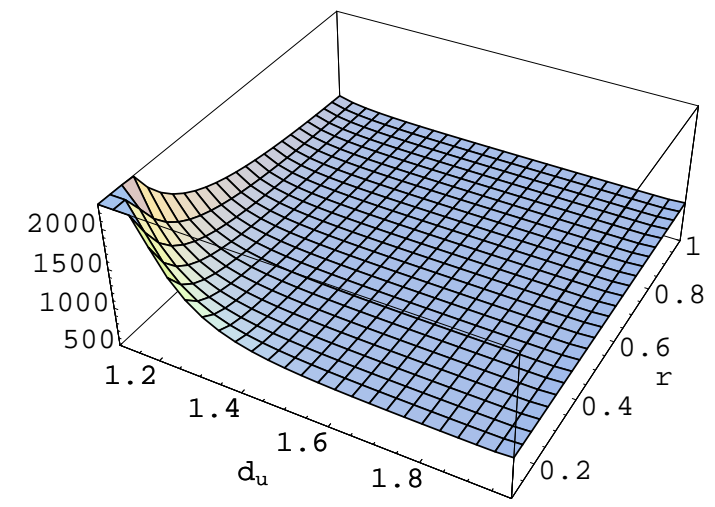

FIG. 4: The upper bound of Higgs mass $m_{h}$ in $\mathrm{GeV}$ (vertical axis) as a function of $d_{\mathcal{U}}$ and $r$.

We will concentrate on $d_{\mathcal{U}}$ between 1 and 2 .

In Fig. 3, we show $\left|a_{0}^{T}\right|$ as functions of the Higgs boson mass $m_{h}$ and the unparticle dimension $d_{\mathcal{U}}$ for several finite values of $r$ in the limit $s>4 m_{h}^{2}$. With a low value for $r$, the allowed region in $m_{h}$ and $d_{\mathcal{U}}$ space is more restrictive than those for larger $r$. This is because that for smaller $r, a_{0}^{u n}$ becomes larger as $d_{\mathcal{U}}$ decreases. To satisfy the unitarity constraint, a large cancelation from the SM contribution is needed and resulting in a larger Higgs boson mass. Fixing $\left|a_{0}^{T}\right|=1$, one can solve an upper bound for the Higgs mass $m_{h}$ as a function of $d_{\mathcal{U}}$ and r. In Fig. 4 we show this upper bound. We see more clearly that for smaller $r$ and $d_{\mathcal{U}}$ much larger Higgs boson mass compared with SM unitarity bound is allowed. When $d_{\mathcal{U}}$ and $r$ become larger, the unparticle effects decreases. The unitarity bound on Higgs boson mass quickly, from above, reaches the SM one.

In summary we have studied unparticle effects on the unitarity constraints from two-body Higgs boson scattering process. We find that the unparticle contribution to the scattering partial wave amplitude can be significant which affect the unitarity constraint on the Higgs 
boson mass. For $d_{\mathcal{U}}$ between 1 and 2, the unparticle contribution can relax the upper bound for Higgs boson mass. For $d_{\mathcal{U}}$ smaller than 1.3 and $r$ smaller than 0.4, the allowed Higgs boson mass can be much larger than that in the SM.

[1] H. Georgi, Phys. Rev. Lett. 98, 221601 (2007); H. Georgi, Phys. Lett. B 650, 275 (2007) arXiv:0704.2457 [hep-ph]].

[2] K. Cheung, W. Y. Keung and T. C. Yuan, Phys. Rev. Lett. 99, 051803 (2007) arXiv:0704.2588 [hep-ph]]; M. Luo and G. Zhu, arXiv:0704.3532 [hep-ph].

[3] C. H. Chen and C. Q. Geng, arXiv:0705.0689 [hep-ph]; G. J. Ding and M. L. Yan, Phys. Rev. D 76, 075005 (2007) arXiv:0705.0794 [hep-ph]]; Y. Liao, Phys. Rev. D 76, 056006 (2007) arXiv:0705.0837 [hep-ph]]; T. M. Aliev, A. S. Cornell and N. Gaur, Phys. Lett. B 657, 77 (2007) arXiv:0705.1326 [hep-ph]]; X. Q. Li and Z. T. Wei, Phys. Lett. B 651, 380 (2007) arXiv:0705.1821 [hep-ph]]; C. D. Lu, W. Wang and Y. M. Wang, Phys. Rev. D 76, 077701 (2007) arXiv:0705.2909 [hep-ph]]; M. A. Stephanov, Phys. Rev. D 76, 035008 (2007) arXiv:0705.3049 [hep-ph]]; N. Greiner, Phys. Lett. B 653, 75 (2007) arXiv:0705.3518 [hepph]]; H. Davoudiasl, Phys. Rev. Lett. 99, 141301 (2007) arXiv:0705.3636 [hep-ph]]; D. Choudhury, D. K. Ghosh and Mamta, arXiv:0705.3637 [hep-ph]; T. M. Aliev, A. S. Cornell and N. Gaur, JHEP 0707, 072 (2007) [arXiv:0705.4542 [hep-ph]]; P. Mathews and V. Ravindran, Phys. Lett. B 657, 198 (2007) arXiv:0705.4599 [hep-ph]].

[4] S. Zhou, arXiv:0706.0302 [hep-ph]; G. J. Ding and M. L. Yan, arXiv:0706.0325 [hep-ph]; C. H. Chen and C. Q. Geng, Phys. Rev. D 76, 036007 (2007) arXiv:0706.0850 [hep-ph]]; Y. Liao and J. Y. Liu, arXiv:0706.1284 [hep-ph]; P. Ball, M. Bander, J. L. Feng, A. Rajaraman and Y. Shirman, arXiv:0706.2677 [hep-ph]; T. G. Rizzo, JHEP 0710, 044 (2007) arXiv:0706.3025 [hep-ph]]; K. Cheung, W. Y. Keung and T. C. Yuan, Phys. Rev. D 76, 055003 (2007) arXiv:0706.3155 [hep-ph]].

[5] S. L. Chen, X. G. He and H. C. Tsai, JHEP 0711, 010 (2007) arXiv:0707.0187 [hepph]]; R. Zwicky, arXiv:0707.0677 [hep-ph]; T. Kikuchi and N. Okada, arXiv:0707.0893 [hepph]; R. Mohanta and A. K. Giri, Phys. Rev. D 76, 075015 (2007) arXiv:0707.1234 [hepph]]; C. S. Huang and X. H. Wu, arXiv:0707.1268 [hep-ph]; A. Lenz, Phys. Rev. D 76, 065006 (2007) arXiv:0707.1535 [hep-ph]]; D. Choudhury and D. K. Ghosh, arXiv:0707.2074 
[hep-ph]; H. Zhang, C. S. Li and Z. Li, arXiv:0707.2132 [hep-ph]; X. Q. Li, Y. Liu and Z. T. Wei, arXiv:0707.2285 [hep-ph]; Y. Nakayama, Phys. Rev. D 76, 105009 (2007) arXiv:0707.2451 [hep-ph]]; N. G. Deshpande, X. G. He and J. Jiang, Phys. Lett. B 656, 91 (2007) arXiv:0707.2959 [hep-ph]]; T. A. Ryttov and F. Sannino, Phys. Rev. D 76, 105004 (2007) arXiv:0707.3166 [hep-th]]; R. Mohanta and A. K. Giri, Phys. Rev. D 76, 057701 (2007) arXiv:0707.3308 [hep-ph]]; A. Delgado, J. R. Espinosa and M. Quiros, JHEP 0710, 094 (2007) arXiv:0707.4309 [hep-ph]].

[6] M. Neubert, arXiv:0708.0036 [hep-ph]; M. x. Luo, W. Wu and G. h. Zhu, arXiv:0708.0671 [hep-ph]; S. Hannestad, G. Raffelt and Y. Y. Y. Wong, arXiv:0708.1404 [hep-ph]; N. G. Deshpande, S. D. H. Hsu and J. Jiang, arXiv:0708.2735 [hep-ph]; P. K. Das, arXiv:0708.2812 [hep-ph]; G. Bhattacharyya, D. Choudhury and D. K. Ghosh, Phys. Lett. B 655, 261 (2007) arXiv:0708.2835 [hep-ph]]; Y. Liao, arXiv:0708.3327 [hep-ph]; D. Majumdar, arXiv:0708.3485 [hep-ph]; A. T. Alan and N. K. Pak, arXiv:0708.3802 [hep-ph]; A. Freitas and D. Wyler, arXiv:0708.4339 [hep-ph]; I. Gogoladze, N. Okada and Q. Shafi, arXiv:0708.4405 [hep-ph].

[7] C. H. Chen and C. Q. Geng, arXiv:0709.0235 [hep-ph]; T. i. Hur, P. Ko and X. H. Wu, arXiv:0709.0629 [hep-ph]; L. Anchordoqui and H. Goldberg, arXiv:0709.0678 [hep-ph]. S. Majhi, arXiv:0709.1960 [hep-ph]; J. McDonald, arXiv:0709.2350 [hep-ph]; M. C. Kumar, P. Mathews, V. Ravindran and A. Tripathi, arXiv:0709.2478 [hep-ph]; S. Das, S. Mohanty and K. Rao, arXiv:0709.2583 [hep-ph]; G. j. Ding and M. L. Yan, arXiv:0709.3435 [hep-ph];

[8] A. B. Balantekin and K. O. Ozansoy, arXiv:0710.0028 [hep-ph]; T. M. Aliev and M. Savci, arXiv:0710.1505 [hep-ph]; K. Cheung, W. Y. Keung and T. C. Yuan, arXiv:0710.2230 [hepph]; E. O. Iltan, arXiv:0710.2677 [hep-ph]; S. L. Chen, X. G. He, X. Q. Li, H. C. Tsai and Z. T. Wei, arXiv:0710.3663 [hep-ph]; I. Lewis, arXiv:0710.4147 [hep-ph]; A. T. Alan, N. K. Pak and A. Senol, arXiv:0710.4239 [hep-ph]; G. L. Alberghi, A. Y. Kamenshchik, A. Tronconi, G. P. Vacca and G. Venturi, arXiv:0710.4275 [hep-th]; S. L. Chen, X. G. He, X. P. Hu and Y. Liao, arXiv:0710.5129 [hep-ph]; O. Cakir and K. O. Ozansoy, arXiv:0710.5773 [hep-ph].

[9] T. Kikuchi and N. Okada, arXiv:0711.1506 [hep-ph]; I. Sahin and B. Sahin, arXiv:0711.1665 [hep-ph]; E. O. Iltan, arXiv:0711.2744 [hep-ph]; A. T. Alan, arXiv:0711.3272 [hep-ph]; K. Cheung, C. S. Li and T. C. Yuan, arXiv:0711.3361 [hep-ph]; R. Mohanta and A. K. Giri, arXiv:0711.3516 [hep-ph]; J. R. Mureika, arXiv:0712.1786 [hep-ph]. Y. Wu and D. X. Zhang, arXiv:0712.3923 [hep-ph]. 
[10] T. Kikuchi, N. Okada and M. Takeuchi, arXiv:0801.0018 [hep-ph]; X. G. He and S. Pakvasa, Phys. Lett. B 662, 259 (2008) arXiv:0801.0189 [hep-ph]]; E. O. Iltan, arXiv:0801.0301 [hep-ph]; C. H. Chen, C. S. Kim and Y. W. Yoon, arXiv:0801.0895 [hep-ph]; V. Bashiry, arXiv:0801.1490 [hep-ph]; J. L. Feng, A. Rajaraman and H. Tu, arXiv:0801.1534 [hep-ph]; K. Cheung, T. W. Kephart, W. Y. Keung and T. C. Yuan, arXiv:0801.1762 [hep-ph]; I. Sahin, arXiv:0801.1838 ; V. Barger, Y. Gao, W. Y. Keung, D. Marfatia and V. N. Senoguz, Phys. Lett. B 661, 276 (2008) arXiv:0801.3771 [hep-ph]]; G. J. Ding and M. L. Yan, Phys. Rev. D 77, 014005 (2008); M. J. Aslam and C. D. Lu, arXiv:0802.0739 [hep-ph]; E. O. Iltan, arXiv:0802.1277 [hep-ph]; B. Sahin, arXiv:0802.1937 [hep-ph]; I. Sahin, arXiv:0802.2818 [hepph]; A. Hektor, Y. Kajiyama and K. Kannike, arXiv:0802.4015 [hep-ph]; Y. Gong and X. Chen, arXiv:0803.3223 [astro-ph]; M. C. Kumar, P. Mathews, V. Ravindran and A. Tripathi, Phys. Rev. D 77, 055013 (2008); F. Sannino, arXiv:0804.0182 [hep-ph]; E. Iltan, arXiv:0804.2456 [hep-ph]; M. C. Kumar, P. Mathews, V. Ravindran and A. Tripathi, arXiv:0804.4054 [hep-ph]; Y. Liao, arXiv:0804.4033 [hep-ph].

[11] P. J. Fox, A. Rajaraman and Y. Shirman, Phys. Rev. D 76, 075004 (2007) arXiv:0705.3092 [hep-ph]].

[12] S. L. Chen and X. G. He, Phys. Rev. D 76, 091702 (2007) arXiv:0705.3946 [hep-ph]].

[13] B. W. Lee, C. Quigg and H. B. Thacker, Phys. Rev. D 16, 1519 (1977).

[14] X. G. He, Phys. Rev. D 61, 036007 (2000) arXiv:hep-ph/9905500.

[15] S. Dawson and S. Willenbrock, Phys. Rev. Lett. 62, 1232(1989); W. Marciano, g. Valencia and S. Willenbrock, Phys. Rev. D40, 1725(1989); L. Durand, J. Johnson and J. Lopez, Phys. Rev. Lett. 64, 1215(1990); Phys. Rev. D45, 3112(1992). 